\title{
Taohuajing reduces oxidative stress and inflammation in diabetic cardiomyopathy through the sirtuin 1/nucleotide-binding oligomerization domain-like receptor protein 3 pathway
}

Rui Yao ${ }^{1,2}$, Yu CaO ${ }^{2}$, Changming Wang ${ }^{1}$, Lu Xu ${ }^{2}$ Xuan Zhang ${ }^{1}$, Yuqing Deng ${ }^{3}$, Feng Li ${ }^{1 *}$ and Siwang Wang ${ }^{2^{*}}$ (D)

\begin{abstract}
Background: Oxidative stress and inflammation promote the development of diabetic cardiomyopathy (DCM). Therefore, inhibiting these processes may show beneficial effects in the treatment of patients with DCM. Taohuajing (THJ) is prepared using Persicae semen (Taoren), Polygonatum sibiricum (Huangjing), and Carthami flos (Honghua) and may have applications in the treatment of DCM. However, the protective effects of THJ have not been thoroughly assessed. Accordingly, in this study, we aimed to investigate the protective effects of $\mathrm{THJ}$ in a model of DCM and further clarify the potential mechanisms.

Methods: A type 2 diabetes mellitus model was generated using male C57BL/6 mice. Echocardiography and histopathology were used to evaluate cardiac function. The expression levels of cytokines were measured using enzyme-linked immunosorbent assays. Western blotting and small interfering RNA were used to evaluate the targets of THJ.
\end{abstract}

Results: Compared with the control group, DCM mice showed cardiac dysfunction, metabolic disorder, fibrosis, and disorganized ultrastructure, and THJ treatment significantly inhibited these changes significantly. THJ treatment also inhibited the production of reactive oxygen species (ROS) and malondialdehyde (MDA), induced the production of glutathione peroxidase (GSH-Px) and superoxide dismutase (SOD), decreased the levels of pro-inflammatory cytokines, and suppressed the activation of the nucleotide-binding oligomerization domain-like receptor protein 3 (NLRP3) inflammasome. These protective effects were abolished by sirtinol, an inhibitor of sirtuin1 (SIRT1).

\footnotetext{
* Correspondence: lifeng@fmmu.edu.cn; wangsiw@fmmu.edu.cn

'Department of Traditional Chinese Medicine, the First Affiliated Hospital, the Air Force Medical University, 127 Changle West Road, Xi'an 710032, Shanxi, China

${ }^{2}$ Department of Chinese Materia Medica and Natural Medicines, School of Pharmacy, the Air Force Medical University, 169 Changle West Road, Xi'an 710032, Shanxi, China

Full list of author information is available at the end of the article
}

(C) The Author(s). 2021 Open Access This article is licensed under a Creative Commons Attribution 4.0 International License, which permits use, sharing, adaptation, distribution and reproduction in any medium or format, as long as you give appropriate credit to the original author(s) and the source, provide a link to the Creative Commons licence, and indicate if changes were made. The images or other third party material in this article are included in the article's Creative Commons. licence, unless indicated otherwise in a credit line to the material. If material is not included in the article's Creative Commons licence and your intended use is not permitted by statutory regulation or exceeds the permitted use, you will need to obtain permission directly from the copyright holder. To view a copy of this licence, visit http://creativecommons.org/licenses/by/4.0/ The Creative Commons Public Domain Dedication waiver (http://creativecommons.org/publicdomain/zero/1.0/) applies to the data made available in this article, unless otherwise stated in a credit line to the data. 
(Continued from previous page)

Conclusions: Overall, THJ protected the heart from hyperglycemia-induced oxidative stress and inflammation in

DCM mice via a mechanism involving SIRT1-mediated antioxidant proteins and suppression of the NLRP3

inflammasome.

Keywords: Taohuajing, Oxidative stress, Inflammation, Diabetic cardiomyopathy, Sirtuin 1, NLRP3

\section{Background}

The incidence of type 2 diabetes mellitus (T2DM) has been increasing worldwide as the rates of obesity, sedentary lifestyle, and environmental alterations have increased; accordingly, it is estimated that half a billion people worldwide will be diagnosed with T2DM by 2030 [1]. Chronic diabetes impairs ventricular function, including macrovascular and microvascular, thereby inducing diabetic cardiomyopathy (DCM), which is one of the main causes of death in patients with T2DM $[2,3]$. In the clinical setting, the goals of treatment include controlling glucose and lipid levels, managing hypertension, and blocking the renin-angiotensin system; however, controlling these parameters alone cannot prevent the pathological changes associated with DCM [3]. Therefore, it is necessary to develop an effective drug that targets multiple pathologies of DCM in order to prevent or delay the development and progression of DCM.

Several mechanisms are likely to contribute to the development of DCM, including cardiac oxidative stress, inflammation, apoptosis, fibrosis, calcium overload, and mitochondrial dysfunction $[4,5]$. The two key metabolic abnormalities, i.e., hyperglycemia and hyperlipidemia, alter the cellular redox status, induce reactive oxygen species (ROS) accumulation, decrease antioxidant protein activities, and disrupt membrane function, resulting in contractile dysfunction within weeks [6-10]. Oxidative stress and inflammation induce cardiac dysfunction, impair insulin signaling, cause heart dysfunction, and decrease endogenous antioxidant levels [11, 12]. Nucleotide-binding oligomerization domain-like receptor proteins (NLRPs), which form a type of inflammasome, have been found to play important roles in the inflammatory process of DCM and other diabetic complications, such as diabetic retinopathy $[13,14]$. Overproduction of ROS in cells will activate the NLRP3 inflammasome and increase inflammatory damage [15]. Thus, drugs or chemicals that can inhibit both oxidative stress and inflammation may have beneficial effects in treating DCM.

Sirtuin 1 (SIRT1) belongs to the sirtuin family and plays important roles in regulating inflammation, oxidative stress, apoptosis, and the cell cycle by deacetylating chromatin histones and multiple transcription factors, including Forkhead transcription factors (FOXOs) and nuclear factor (NF) $-\kappa B$ [16-18]. FOXO3a, a FOXO family member expressed in the heart, transcriptionally regulates the expression of manganese superoxide dismutase (MnSOD), which protects cells from oxidative stress [19]. Additionally, SIRT1 interacts with NF- $\kappa B$ by inhibiting the transcription of p65, thereby blocking the expression of inflammatory factors [20,21]. Accordingly, regulating the expression and/or the activity of SIRT1 may be useful to manage inflammation and oxidative stress induced by hyperglycemia and hyperlipidemia in DCM.

Traditional Chinese medicine (TCM), which is based on ancient philosophies regarding the use of herbs and plants, has been shown to be effective in treating DCM, with fewer side effects [22, 23]. Taohuajing (THJ) is composed of Persicae semen (Prunus persica (L.) Batsch, Taoren), Carthami flos (safflower, Carthamus tinctorius (L.), Honghua), and Polygonatum sibiricum(F.) (Huangjing), based on the TCM theory. The pair of Persicae semen and Carthami flos is a classical medicinal pair used in many well-known prescriptions, including Taohongsiwutang, Buyanghuanwutang, and Taorenchengqitang [24, 25]. Extracts of Carthami flos have antidiabetic effects in an alloxan-induced diabetic model in rats and exhibit antioxidative effects in scavenging free radicals and enhancing endogenous antioxidant activity in rat cardiac microvascular endothelial cells [26, 27]. Moreover, Persicae semen can inhibit macrovascular fibrosis by regulating the AKT pathway in streptozotocin (STZ)induced diabetic rats [28]. Polygonatum sibiricum has many physiological effects, including antioxidant, antiaging, anti-inflammatory, hypolipidemic, and heart protective effects, and has been used in the treatment of diabetes mellitus, exhibiting excellent antidiabetic activity [29-31]. The total saponins from Polygonatum sibiricum can promote glycogenesis and glucose utilization in peripheral tissue, thereby controlling blood glucose levels [32]. Additionally, polysaccharides from Polygonatum sibiricum also show protective effects by inhibiting the binding of glycosylated end-products in cardiac tissues in diabetic model mice [33].

Although these individual medicines have been shown to have antidiabetic activities and cardioprotective effects, no studies have reported whether the combination of these three medicines can be used to treat diabetic cardiomyopathy. Therefore, in the current study, we 
assessed the protective effects of THJ on a DCM model induced by a high-fat diet (HFD) and STZ.

\section{Methods \\ Materials}

Persicae semen (Taoren) was obtained from Hebei province, China. Carthami flos (Honghua) was purchased from Xinjiang province, China. Polygonatum sibiricum (Huangjing) was purchased from Shaanxi, China. 2,3,5Triphenyltetrazolium chloride (TTC) was purchased from Sigma-Aldrich (St. Louis, MO, USA). Kits for the detection of insulin, SOD, malondialdehyde (MDA), glutathione peroxidase (GSH-Px), and caspase 3 were obtained from Nanjing Jiancheng Bioengineering Institute (Nanjing, China). Dihydroethidium (DHE) was obtained from Invitrogen (CA, USA). The primary antibodies against NPL65 (cat. no. 13158), cleavedcaspase 3 (cat. no. 9664), and cleaved-caspase 9 (cat. no. 20750) were obtained from Cell Signaling Technologies (MA, USA). The NAD+/NADH assay kit was purchased from Abcam (Carlsbad, CA, USA). Anti-acetylated (Ac)FOXO3 and anti-SIRT1 primary antibodies were obtained from Santa Cruz Biotechnology (Santa Cruz, CA, USA). All other materials used in this study were commercially available.

\section{Preparation of THJ}

Persicae semen (300 g), Carthami flos (180 g), and Polygonatum sibiricum $(300 \mathrm{~g})$ were obtained (The weight ratio of Persicae semen: Carthami flos: Polygonatum sibiricum is 5:3:5) and crushed into small pieces, and the concoction extracted with deionized water $(1: 10, \mathrm{w} / \mathrm{v})$ twice for $30 \mathrm{~min}$ each. Filtrates from the two batches were combined, concentrated, and spray dried.

\footnotetext{
Animals

C57BL/6 mice (8-10 weeks old, $23-25 \mathrm{~g}$ ) were purchased from the Animal Experimental Center of the Air Force Medical University. Mice were housed with free access to water and standard food in an experimental animal room with a 12/12-h light/dark cycle, temperature maintained at $22 \pm 2{ }^{\circ} \mathrm{C}$, and relative humidity of $61-65 \%$. The animals were provided with a standard laboratory diet and water and were checked every day for any health issues. All protocols in this study were approved by the Ethics Committee for Animal Experimentation of the Air Force Medical University and performed according to the National Institute of Health Guide for the Care and Use of Laboratory Animals (NIH Publications No. 80-23) revised in 1996 and the Animal Research Reporting In Vivo Experiments guidelines.
}

\section{DCM model and groups}

After acclimation for 1 week, mice in the diabetes groups were fed an HFD containing $45 \% \mathrm{kcal}$ from fat for 4 weeks and then intraperitoneally injected with $60 \mathrm{mg} / \mathrm{kg}$ STZ (Sigma) dissolved in citrate buffer ( $\mathrm{pH} 4.5)$ for 3 days. The same volume of citrate buffer was intraperitoneally injected into mice in the control group. After 2 weeks, fasting-blood glucose (FBG) was measured using a BREEZE2 meter (Bayer, Mishawaka, IN, USA). Mice with an FBG of more than $11.1 \mathrm{mM}$ were considered diabetic and were used in further studies. The DCM model was induced according to previous studies [34]. Animals were randomly assigned to five groups: control group (Con, $n=15$ ), DCM group (DCM, $n=15$ ), lowdose THJ-treated DCM group $(0.125 \mathrm{~g} / \mathrm{kg} /$ day, THJ-L, $\mathrm{n}$ = 15), medium-dose THJ-treated DCM group $(0.25 \mathrm{~g} /$ $\mathrm{kg} /$ day, $\mathrm{THJ}-\mathrm{M}, \mathrm{n}=15$ ), and high-dose THJ-treated DCM group $(0.5 \mathrm{~g} / \mathrm{kg} /$ day, $\mathrm{THJ}-\mathrm{H}, \mathrm{n}=15)$. Mice in the THJ groups were administered $\mathrm{THJ}$ by gavage at doses of $0.125,0.25$, or $0.5 \mathrm{~g} / \mathrm{kg} /$ day for 12 weeks. Mice in the DCM groups were administered the same volume of vehicle by gavage. Mice in both the DCM and THJ groups were given free access to an HFD, and mice in the Con group were fed standard chow. Because the formula for THJ was modified based on the Tonghongsiwu decoction, the dosages of $\mathrm{THJ}$ in animal experiments were set according to the dosage of Tonghongsiwu decoction and our preliminary experiment $[35,36]$. After all the experiments were completed, all mice were euthanized with $150 \mathrm{mg} / \mathrm{kg}$ pentobarbital sodium through intraperitoneal injection under standard protocols. All efforts were made to minimize suffering.

\section{Determination of cardiac function}

At the end of the experiment, mice were re-anesthetized using $1.0 \%$ isoflurane (the heart rate was maintained at 400-500 bpm), and cardiac function was determined by transthoracic echocardiography with a Visual-Sonics Vevo 770 ultrasound system (Toronto, Canada). Left ventricular fractional shorting (LVFS), left ventricular ejection fraction (LVEF), left ventricular end-diastolic volume (LVEDV), and left ventricular end-systolic volume (LVESV) were determined using computer algorithms.

\section{Hematoxylin-eosin (HE) and Masson's trichrome staining} After measuring cardiac function, hearts were collected, and $4 \%$ paraformaldehyde solution was used to fix the heart tissues. Heart tissues were then embedded in paraffin and sectioned at $5 \mu \mathrm{m}$ thickness. Sections were incubated with 1\% TTC solution (pH 7.4) for $15 \mathrm{~min}$ at $37^{\circ} \mathrm{C}$. Extracellular collagen deposition in the heart tissue was examined by Masson's trichrome staining. 


\section{Determination of biochemical indexes}

Blood samples were collected from mice after sacrifice, centrifuged at $2500 \times \mathrm{g}$ for $15 \mathrm{~min}$ at $4{ }^{\circ} \mathrm{C}$ to collect the supernatants, and stored at $-80^{\circ} \mathrm{C}$. Relative levels of tumor necrosis factor- $\alpha$ (TNF- $\alpha$ ), interleukin (IL)-6, GSH-Px, IL-1 $\beta$, SOD, and MDA were determined using enzyme-linked immunosorbent assay (ELISA) kits, according to the manufacturer's protocols. Insulin levels were measured using an insulin assay kit, and the insulin sensitivity index (ISI) was calculated as $1 /$ (blood glucose $x$ serum insulin). NAD+ and NADH levels were measured using an NAD+/NADH assay kit, and the NAD+/ NADH ratio was calculated.

Transmission electron microscopy (TEM) analyses of heart tissues

TEM was used to detect changes in the ultrastructure morphology of myofibrils and mitochondria. Tissues with a thickness of $60-64 \mathrm{~nm}$ and pieces measuring 1 $\mathrm{mm}^{3}$ were cut from the left ventricle of mouse heart tissue, and sections were fixed with $2.5 \%$ glutaraldehyde ( $\mathrm{pH}$ 7.4) for $2 \mathrm{~h}$. Sections were dehydrated in alcohol, embedded, polymerized, stained with uranyl acetate and lead citrate, and then observed by TEM (JEM-1200EX; Japan).

\section{ROS detection}

ROS in myocardial frozen sections was detected by DHE staining, as previously reported [37, 38]. After staining with DHE and 4',6-diamidino-2-phenylindole, tissue sections were observed using an Olympus FV1000 laser confocal microscope (Olympus, Japan). The fluorescence density value was determined using an Image-Pro Plus (Version 6.0, Media Cybernetics, USA).

\section{Western blotting}

RIPA lysis buffer was prepared with $1 \%$ phenylmethylsulfonyl fluoride and $1 \%$ protease inhibitor cocktail. Heart tissues were collected, rinsed, homogenized with RIPA lysis buffer at $4{ }^{\circ} \mathrm{C}$ for $30 \mathrm{~min}$, and centrifuged to collect supernatants. Protein concentrations were detected using a BCA protein assay kit (Nanjing Jiancheng) according to the manufacturer's instructions. Total proteins $(30 \mu \mathrm{g})$ were loaded and separated by sodium dodecyl sulfate polyacrylamide gel electrophoresis on $10 \%$ gels. Polyvinylidene difluoride membranes were activated with methyl alcohol, and the proteins were then transferred onto the membranes. Next, the membranes were blocked with $5 \%$ nonfat milk for $1 \mathrm{~h}$ at $37^{\circ} \mathrm{C}$. After washing, the membranes were incubated with primary antibodies targeting NLRP3, thioredoxin interacting protein (TXNIP), caspase $1 \mathrm{p} 20$, IL-1 $\beta$ p17, apoptosisassociated speck-like protein containing a CARD (ASC), SIRT1, Ac-FOXO3a, Ac-SOD2 overnight at $4{ }^{\circ} \mathrm{C}$. After rinsing and incubating with secondary antibodies at $37^{\circ} \mathrm{C}$ for $1 \mathrm{~h}$, the bands were detected using enhanced chemiluminescence (Pierce). The bands were scanned using a Bio-Rad Chemidoc XR+ Gel Imaging System, and the optical densities were quantified using a Bio-Rad Image Analysis System. $\beta$-Actin was used as the internal control.

\section{Statistical analysis}

Statistical analysis was performed using Graph Pad Prism 5.0. Values are shown as means \pm standard deviations from at least three different experiments. Differences among experimental groups were analyzed by one-way analysis of variance, followed by Tukey's multiple comparisons tests. Results with $P$ values of less than 0.05 were considered statistically significant.

\section{Results}

THJ prevented systemic metabolic abnormities in diabetic mice

As shown in Table 1, levels of glucose and lipids (triglycerides [TGs] and total cholesterol [TC]) were both significantly increased in diabetic mice $(P<0.05)$. Additionally, the HFD and STZ treatment significantly decreased insulin levels in the blood $(P<0.05)$ and impaired insulin sensitivity, indicating that diabetes induced systemic metabolic abnormities in diabetic mice. After treatment with THJ, glucose and lipid levels were decreased, and insulin sensitivity was improved in a dose-dependent manner. Notably, $0.5 \mathrm{~g} / \mathrm{kg}$ THJ showed the best effects. These results indicated that $\mathrm{THJ}$

Table 1 Effects of THJ on systemic metabolic abnormalities in diabetic mice

\begin{tabular}{llllll}
\hline & Control & DCM & THJ-L & THJ-M & THJ-H \\
\hline Blood glucose $(\mathrm{mM})$ & $3.67 \pm 0.84$ & $24.91 \pm 2.71^{\# \#}$ & $20.14 \pm 1.76$ & $18.32 \pm 1.15^{*}$ & $18.5 \pm 1.08^{*}$ \\
TG (mM) & $0.53 \pm 0.07$ & $4.12 \pm 0.52^{\# \#}$ & $3.81 \pm 0.43$ & $3.15 \pm 0.38^{* *}$ & $2.14 \pm 0.31^{* *}$ \\
TC (mM) & $1.74 \pm 0.33$ & $5.13 \pm 0.57^{\# \#}$ & $4.04 \pm 0.51^{*}$ & $3.15 \pm 0.44^{* *}$ & $2.47 \pm 0.39^{* *}$ \\
INS (mM) & $12.72 \pm 1.36$ & $7.15 \pm 0.87^{\# \#}$ & $7.31 \pm 0.91$ & $7.7 \pm 1.03^{*}$ & $8.52 \pm 1.15^{* *}$ \\
ISI & $0.022 \pm 0.003$ & $0.0057 \pm 0.00065^{\# \#}$ & $0.007 \pm 0.0013$ & $0.0076 \pm 0.0009^{*}$ & $0.0102 \pm 0.0024^{* *}$ \\
\hline
\end{tabular}

Data are shown as means \pm standard deviations, $n=6$-8/group

${ }^{\# \#} P<0.01$ versus control group, ${ }^{*} P<0.05,{ }^{* *} P<0.01$ versus the DCM group 
alleviated the systemic metabolic abnormalities induced by HFD and STZ treatment in diabetic mice.

THJ ameliorated diabetes-induced myocardial dysfunction To investigate whether THJ protected against diabetesinduced myocardial dysfunction, cardiac function was examined in DCM model mice. LVFS and LVEF, two very important indicators for cardiac function, were recorded by echocardiography. As shown in Fig. 1a-c, compared with control mice, LVEF and LVFS were significantly decreased in the DCM group; however, THJ treatment significantly increased LVEF and LVFS. Additionally, THJ treatment reduced the HFD+STZ-induced increases in LVEDV (Fig. 1d) and LVESV (Fig. 1e) in DCM mice. These results implied that THJ treatment significantly alleviated myocardial dysfunction in DCM mice.

THJ ameliorated diabetes-induced myocardial dysfunction in DCM model mice

Myocardial dysfunction is partly induced by myocardial fibrosis, which results in disorders of the pathogenic structure and remodeling of hearts. To study whether THJ affected myocardial fibrosis, we carried out Masson staining. As shown in Fig. 2a, compared with the Con group, interstitial and perivascular Masson staining demonstrated that collagens were significantly increased in DCM mouse hearts. Compared with the DCM group, fibrosis and collagen contents were both decreased in interstitial and perivascular areas.

HE staining results showed that there were major pathogenic structural changes in longitudinal and transverse sections, and cardiomyocytes and hypertrophic myocardium were significantly disrupted in the hearts of DCM model mice. In the THJ treatment group, pathological structural changes were markedly alleviated compared with those in the DCM group (Fig. 2b). TEM was also used to observe microstructure changes. In control mice, typical symmetric myofibrils, well-organized sarcomeres, and mitochondria were observed (Fig. 2c). However, tissues from DCM hearts showed serious injury to the left ventricular ultrastructure, including ruptured and irregular myofibrils, swollen mitochondria, and coalescence of irregular mitochondria. In the THJ treatment groups, these changes were significantly alleviated, and the cardiac ultrastructure in the high-dose treatment group was similar to that in the normal group.

\section{THJ ameliorated diabetes-induced oxidative stress in DCM model mice}

ROS levels in heart tissues were detected by DHE staining. As shown in Fig. 3a and b, compared with the Con group,

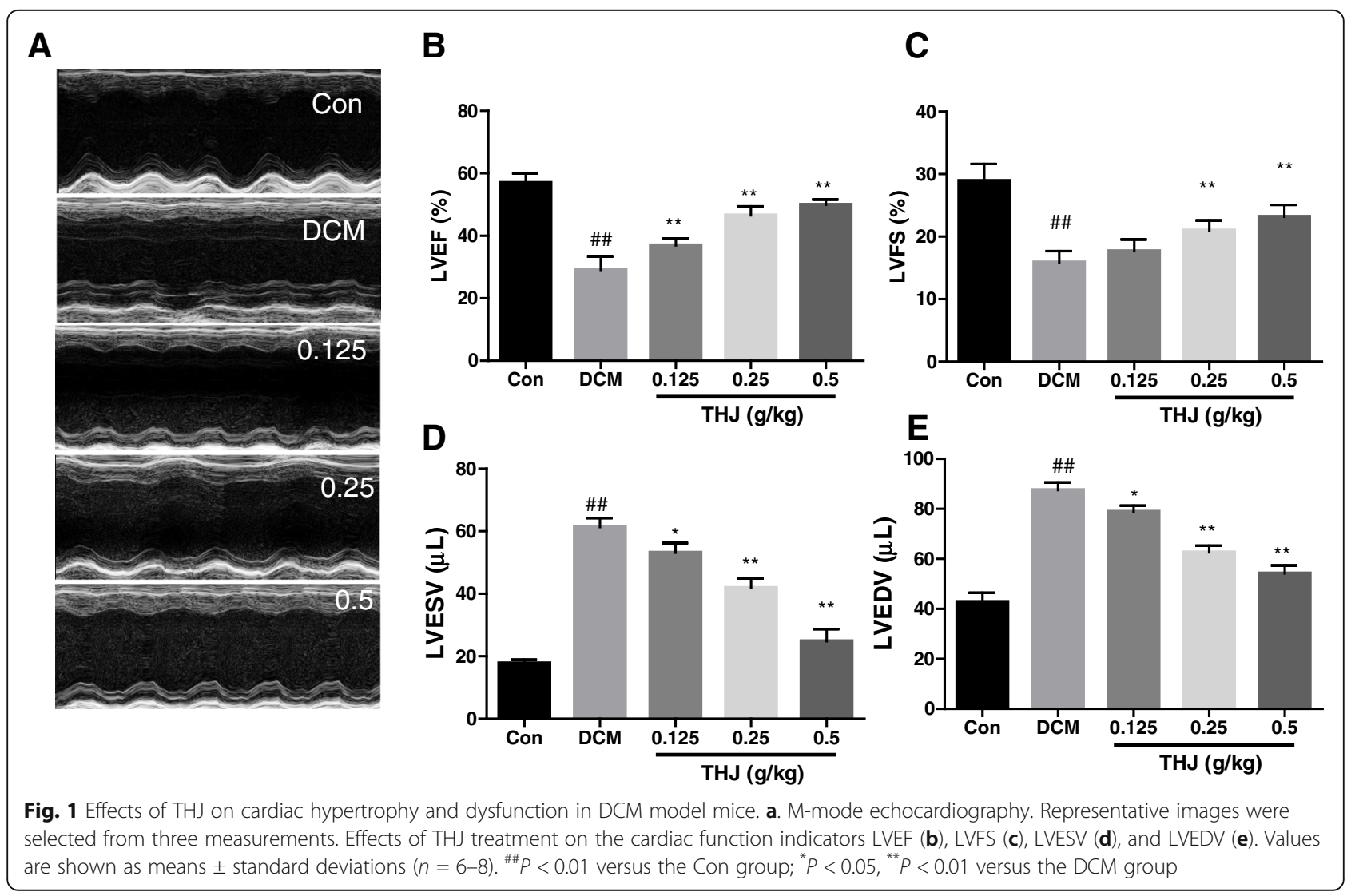




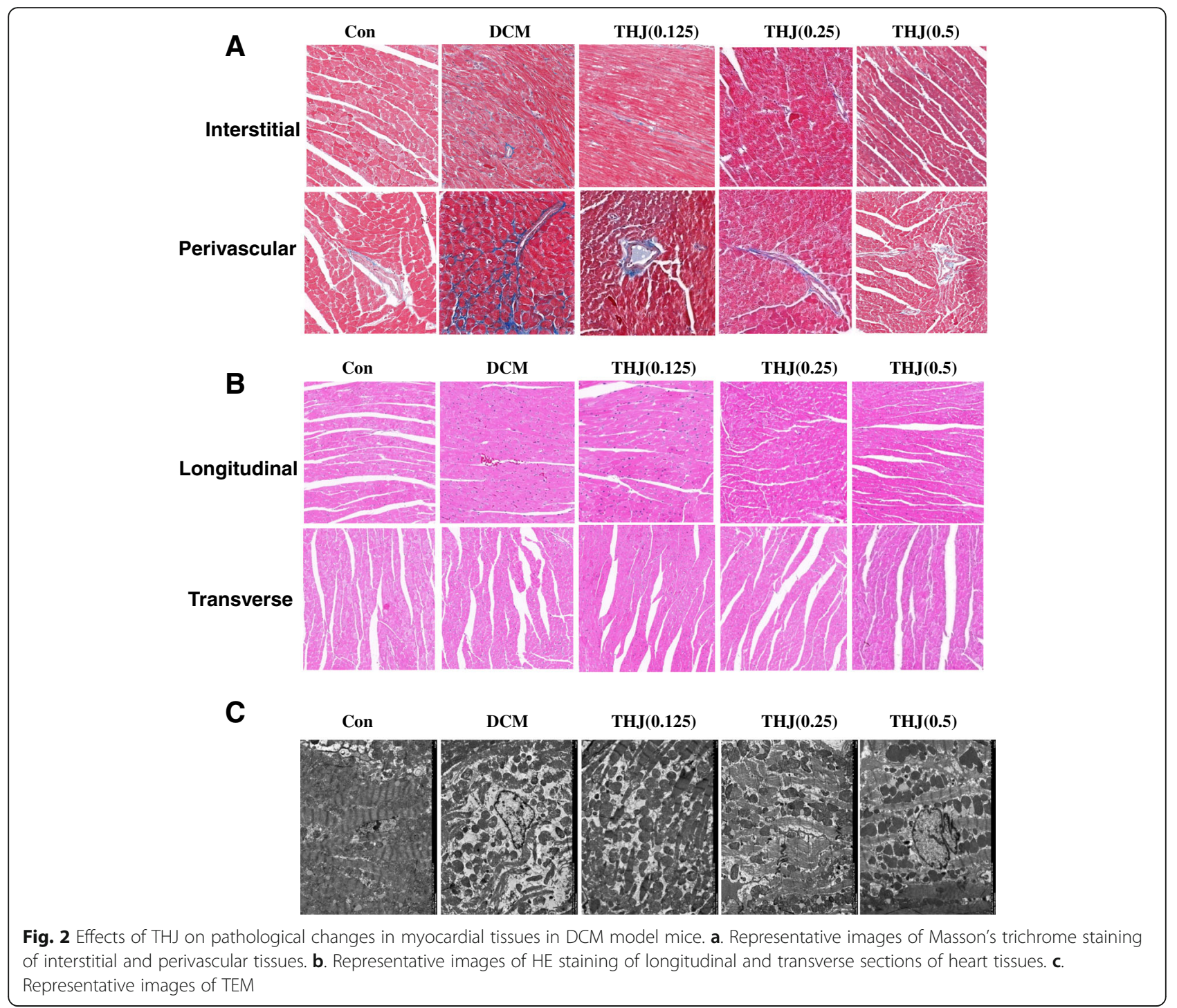

the intensity of DHE fluorescence was significantly increased in the DCM group. Treatment with THJ significantly deceased the intensity of DHE fluorescence, indicating that $\mathrm{THJ}$ inhibited the generation of ROS in the heart. Moreover, MDA levels were significantly elevated in the DCM group and decreased in the THJ treatment groups. Notably, the enzymatic activities of SOD and GSH-Px were significantly decreased in the DCM group (Fig. 3c and d). However, THJ significantly elevated the enzymatic activities of SOD (Fig. 3c, $P<0.01$ ) and GSHPx (Fig. 3d, $P<0.01$ ) in a dose-dependent manner. Overall, these results indicated that THJ treatment inhibited oxidative stress in DCM model mice.

\section{THJ inhibited inflammation and NLRP3 inflammasome activation in DCM model mice}

Next, to evaluate the inflammatory response in DCM model mice, serum levels of inflammatory cytokines (i.e.,
TNF- $\alpha$, IL-6, and IL-1 $\beta$ ) were measured. As shown in Fig. 4, the levels of TNF- $\alpha$ (Fig. 4a), IL-6 (Fig. 4b), and IL-1 $\beta$ (Fig. 4c) were significantly increased in the DCM group compared with those in the Con group. In the THJ treatment groups, serum levels of inflammatory cytokines were significantly decreased in a dose-dependent manner.

To assess whether the NLRP3 inflammasome was activated by diabetes, the expression levels of NLRP3 and related proteins in left ventricular tissue were measured by polymerase chain reaction (PCR) and western blotting. The mRNA levels of caspase-1 (Fig. 4d), NLRP3 (Fig. 4e), TXNIP (Fig. 4f), ASC (Fig. 4g), and $I L-1 \beta$ (Fig. $4 \mathrm{~h}$ ) in the DCM group were significantly higher than those in the Con group. Additionally, protein levels of NLRP3 and related proteins were also higher than those in the Con group (Fig. 5). Compared with the DCM group, the mRNA and protein levels of NLRP3 and 

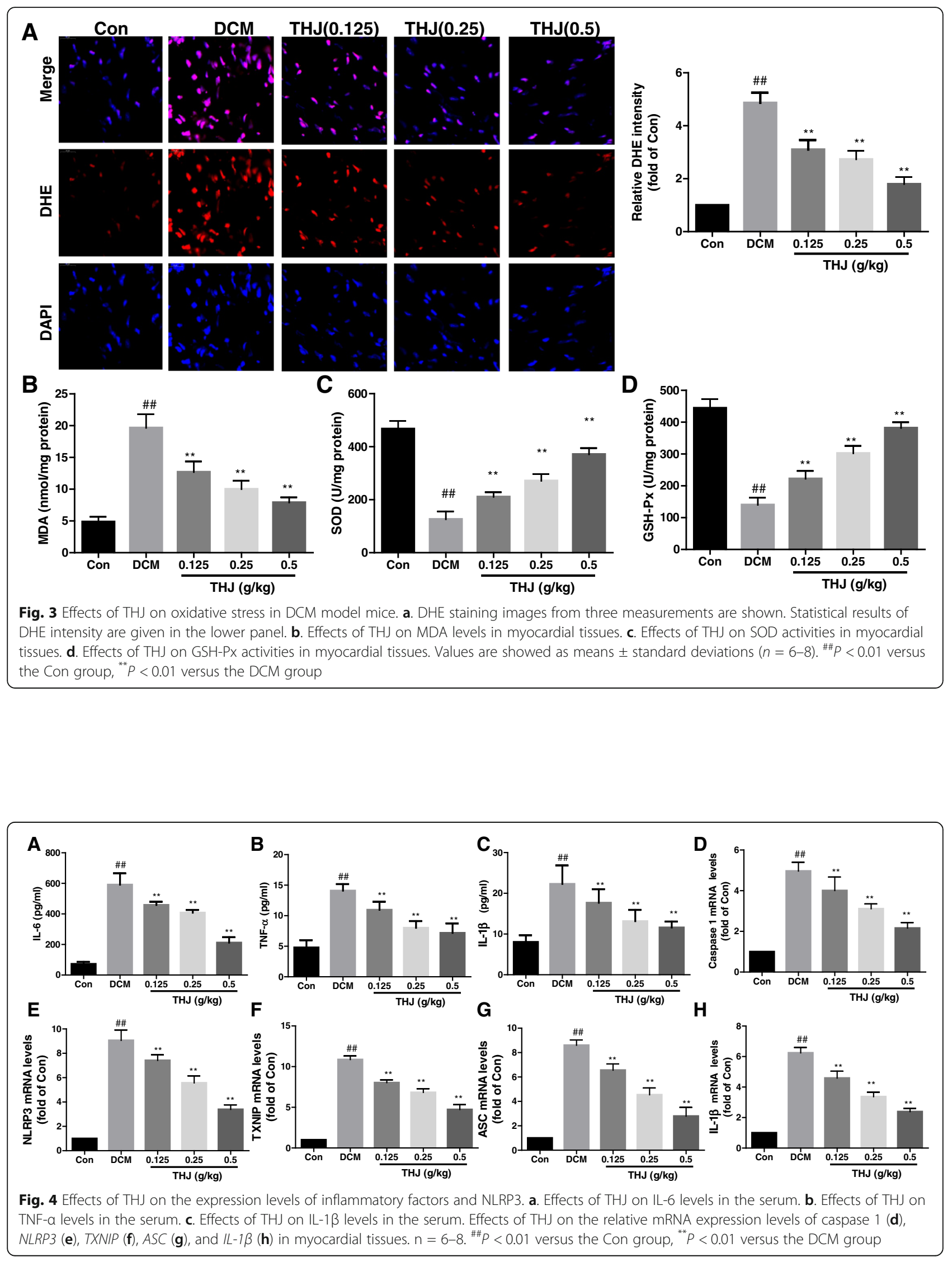


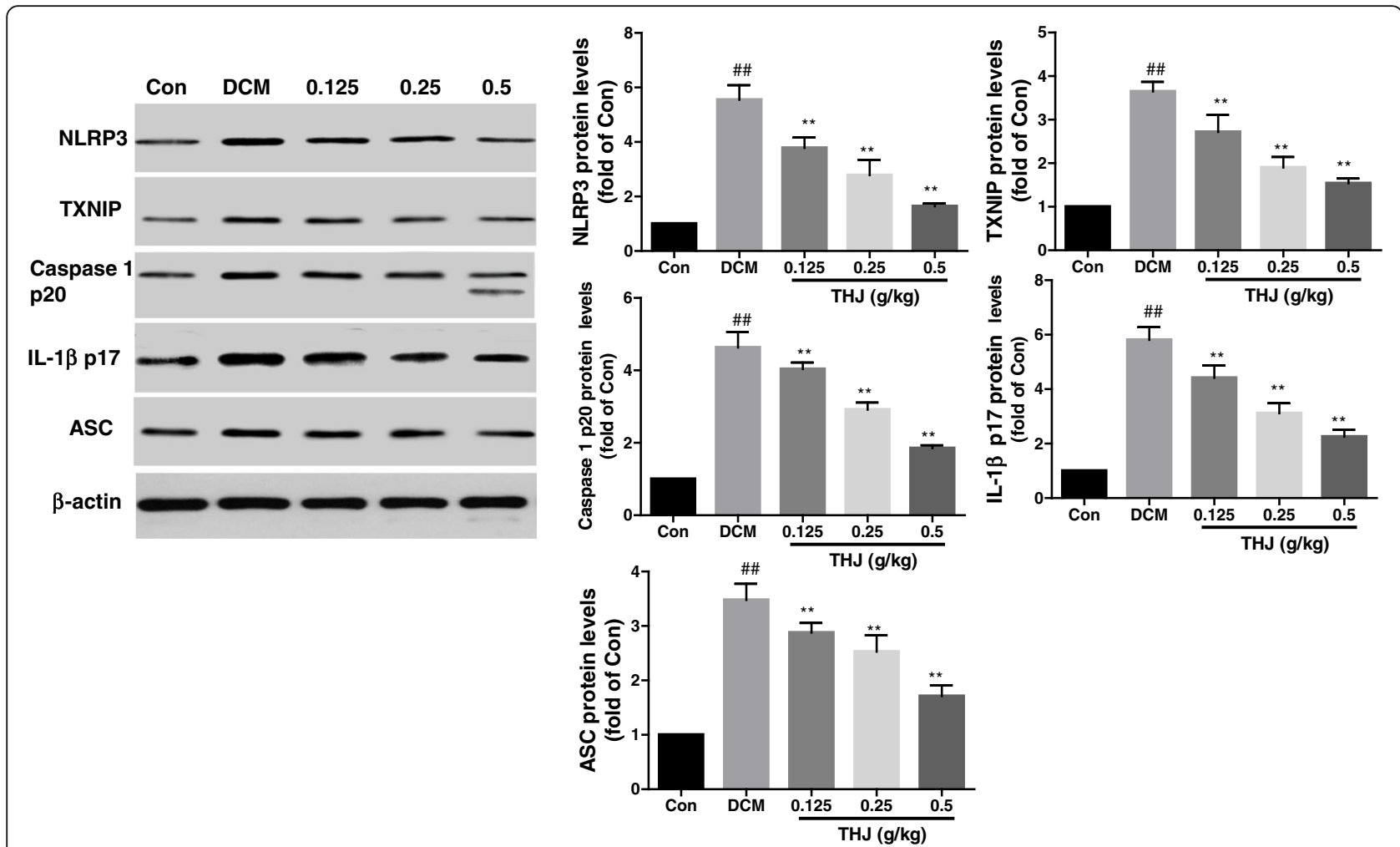

Fig. 5 Effects of THJ on the expression levels of NLRP3-related proteins. Representative blots of NLRP3, TXNIP, caspase 1 p20, IL-13 p17, and ASC are shown on the left. Histograms show quantitative expression levels of these targets on the right. Expression levels were normalized to the expression of $\beta$-actin. Values are shown as means \pm standard deviations from three different experiments. ${ }^{\# \#} P<0.01$ versus the Con group, ${ }^{* *} P<$ 0.01 versus the DCM group

related proteins (i.e., caspase-1, TXNIP, ASC, and IL-1 $\beta$ ) were significantly reduced in the THJ treatment groups.

\section{THJ inhibited oxidative stress by activating SIRT1}

Next, we assessed activation of SIRT1 signaling pathways. In the DCM group, the NAD+/NADH ratio was lower than that in the Con group (Fig. 6a), whereas THJ treatment significantly elevated the $\mathrm{NAD}+\mathrm{NADH}$ ratio in a dose-dependent manner. SIRT1 and its two main downstream proteins, FOXO3a and SOD2, were detected. As shown in Fig. 6b, SIRT1 expression levels were significantly inhibited, and Ac-SOD2 and AcFOXO3a levels were both increased in the DCM group compared with those in the Con group. Interestingly, $\mathrm{THJ}$ treatment dramatically reversed these changes compared with the DCM group. To confirm the role of SIRT1 in mediating the effects of THJ treatment, mice were treated with the SIRT1 specific inhibitor sirtinol. As shown in Fig. 6c, sirtinol treatment decreased protein expression levels of SIRT1. Compared with the THJ group, the THJ+sirtinol group showed higher ROS (Fig. 6d) and MDA levels (Fig. 6e) and lower SOD levels (Fig. 6f), indicating that the inhibitory effects of THJ on oxidative stress were abolished by sirtinol.
Inhibition of SIRT1 suppressed THJ-mediated antiinflammatory activity

To further confirm the roles of SIRT1 in THJmediated anti-inflammatory activity in DCM model mice, mice were treated with sirtinol for 12 weeks. As shown in Fig. 7a, sirtinol abolished the inhibitory effects of THJ on NLRP3 inflammasome activation compared with that in the THJ group. Additionally, we also found that sirtinol treatment blocked the inhibition of TNF- $\alpha$ and IL-6 expression in DCM model mice (Fig. $7 \mathrm{~b}$ and c).

\section{Discussion}

T2DM is characterized by hyperglycemia; disruption of carbohydrate, fat, and protein metabolism; and insulin resistance. Collectively, these factors accelerate the development of cardiomyopathy [39]. DCM is one of the most serious complications of advanced T2DM [40] and is characterized by myocardial dilatation and hypertrophy as well as a decrease in the systolic and diastolic functions of the left ventricle. Additionally, DCM can directly lead to cardiac dysfunction and eventually progress to heart diseases, such as heart failure, arrhythmia, and cardiogenic shock [41, 42]. However, therapeutic 


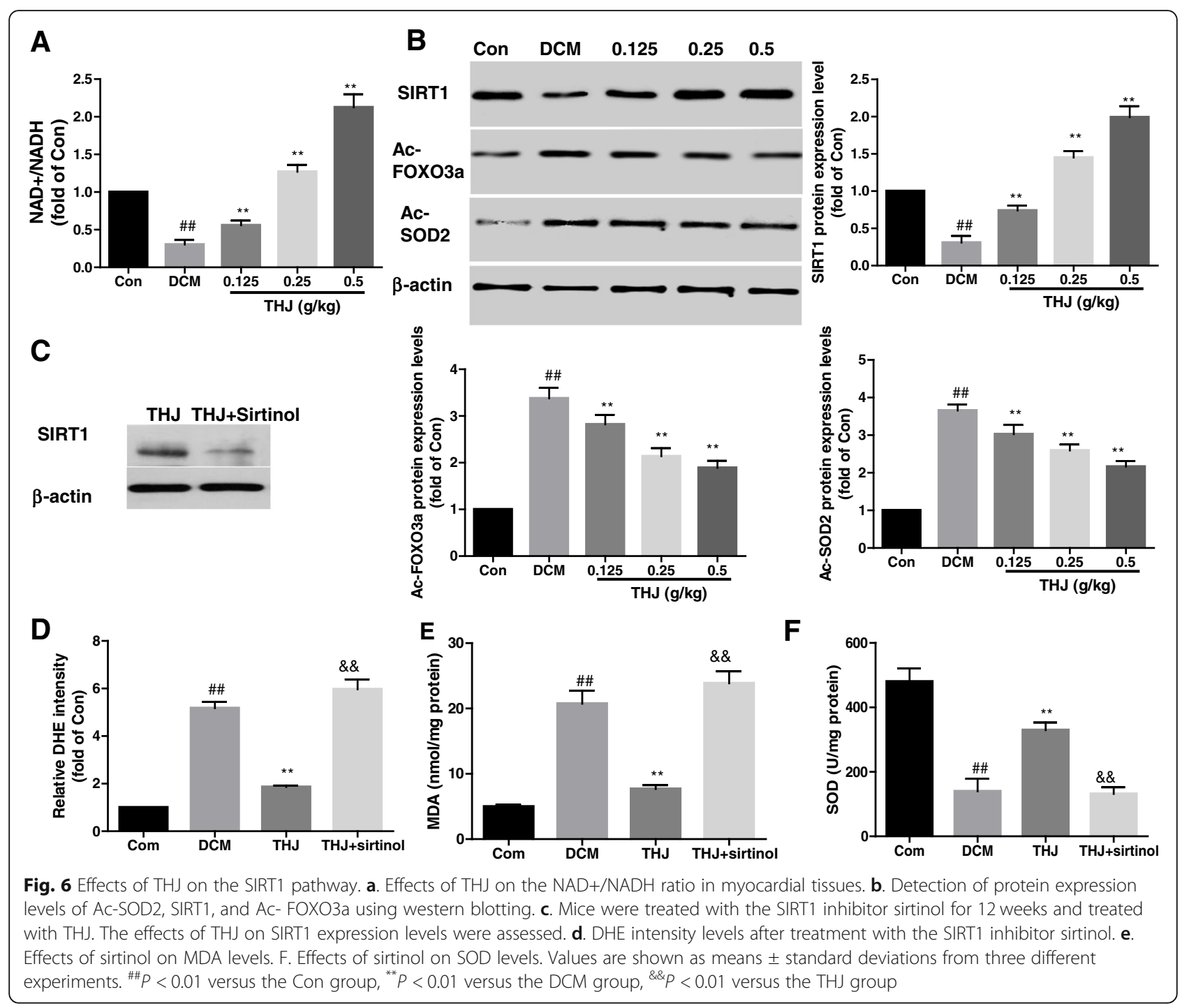

drugs for DCM are not efficacious in the clinical setting; thus, new medicines or treatment are needed.

TCM has been used for thousands of years and has been shown to be beneficial for preventing and treating DCM with minimum side effects [43, 44]. THJ is a TCM developed from Taohongsiwutang and has been used to treat diabetes and diabetes-related complications [45]. This prescription has putative functions in promoting blood circulation to remove blood stasis and in dredging the channels of humans, based on TCM theory [46-48]. However, the effects of THJ on DCM have not been reported. In this study, we aimed to investigate the effects of THJ on DCM induced by HFD and STZ and to explore the potential mechanisms. We expect the results could provide reference information to promote the rational use of $\mathrm{THJ}$ in the clinic.

In this study, we found that blood glucose, TG, and TC levels were elevated, and insulin resistance was induced by HFD and STZ treatment. Blood glucose and lipid levels were both significantly decreased in a dosedependent manner after THJ treatment for 12 weeks, and insulin resistance was significantly alleviated, indicating that THJ alleviated systemic metabolic disorders in diabetic mice. Additionally, diabetes induced heart dysfunction, myofibril and mitochondrial dysfunction, and cardiac ultrastructure disorganization in DCM model mice. Importantly, THJ treatment significantly ameliorated heart dysfunction and reduced pathological alterations in myofibrils and mitochondria, indicating that THJ protected cardiac muscle from structural changes in DCM model mice.

The main characteristics of diabetes are chronic hyperglycemia and hyperlipidemia, and these two factors contribute to the development of cardiac dysfunction [49]. Increased glucose and lipid metabolism enhances the production of ROS by mitochondria and destroys the 


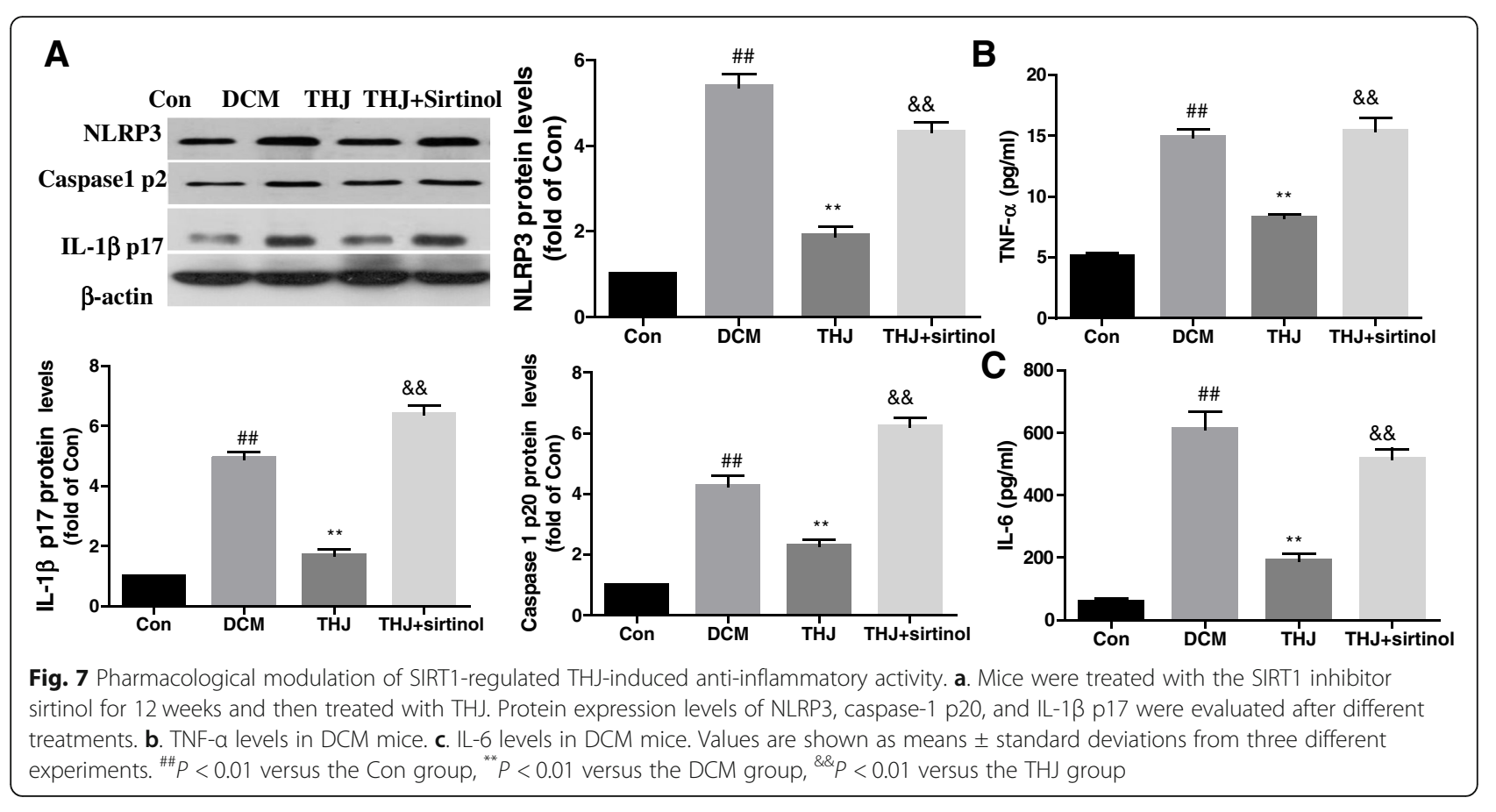

antioxidant system, which induces oxidative stress [42]. ROS cause protein, DNA, and RNA damage and induce cardiomyocyte apoptosis, resulting in a reduction in myocardial contractility and leading to myocyte fibrosis [40]. Consistent with previous studies, we found that ROS levels were elevated in DCM heart tissues and that the levels of antioxidant proteins (GSH-Px and SOD) were decreased, whereas MDA levels were increased. However, following treatment with THJ, ROS and MDA levels were reduced, and antioxidant protein levels were elevated in a dose-dependent manner, indicating that $\mathrm{THJ}$ protected the heart from oxidative stress in DCM model mice.

In the diabetic state, chronic hyperglycemia induces the secretion of pro-inflammatory cytokines and leads to persistent inflammation, which exacerbates or aggravates myocardial injury [50]. In the current study, the proinflammatory cytokines TNF- $\alpha$, IL- 6 , and IL- $1 \beta$ were significantly upregulated in DCM model mice. Many studies have shown that the NLRP3 inflammasome can regulate IL-1 $\beta$ activation in diabetes [51-53]. Hyperglycemia activates the NLRP3 inflammasome, accompanied by TXNIP activation, which inhibits the expression of thioredoxin [54]. The NLRP3 inflammasome contains NLRP3, caspase recruitment domain (ASC), and caspase-1 [55]. Once activated by some stimulation, NLRP3 combines with its adaptor ASC, catalyzes procaspase- 1 to form an active caspase- 1 p10/20 tetramer, which processes pro-IL-1 $\beta$ into its mature form, IL- $1 \beta$ $[56,57]$. In this study, we demonstrated that the mRNA and protein levels of NLRP3 and related proteins were increased in the DCM group, indicating that the NLRP3 inflammasome was activated in diabetic myocardial tissues. In the THJ treatment groups, the expression levels of NLRP3, TXNIP, caspase-1 p10, and IL- $1 \beta$ p17 were significantly decreased in a dose-dependent manner. Thus, our results indicated that THJ inhibited activation of the NLRP3 inflammasome, which was induced by diabetes.

Antioxidant proteins and NLRP3 are regulated by SIRT1. Therefore, to further understand the antioxidative and anti-inflammatory mechanisms of THJ, we evaluated the roles of SIRT1. SIRT1 plays some important roles in regulating the stress response, such as oxidative stress, inflammation, and apoptosis [58]. SIRT1 induces deacetylation of FOXO3a, thereby increasing the ability of FOXO3a to regulate antioxidant proteins (e.g., SOD and CAT) and DNA repair $[59,60]$. Additionally, SIRT1 induces deacetylation of SOD2 directly and increases the antioxidative capacity of SOD2 [61]. In the current study, SIRT1 protein expression levels and downstream proteins (Ac-FOXO3a and Ac-SOD2) were detected to evaluate the regulatory effects of THJ on the SIRT1 pathway. Interestingly, $\mathrm{THJ}$ treatment increased the levels of SIRT1, Ac-FOXO3a, and Ac-SOD2 and enhanced the NAD+/NADH ratio, which were decreased in DCM model mice. A previous study showed that SIRT1 has negative regulatory effects on NLRP3 activation in vascular endothelial cells [62]. In this study, we found that SIRT1 expression levels were suppressed by treatment with the SIRT1 inhibitor sirtinol, accompanied by enhanced NLRP3 inflammasome activation and 
oxidative stress. These results indicated that the mechanisms through which $\mathrm{THJ}$ suppressed oxidative stress and the NLRP3 inflammasome were SIRT1 dependent.

\section{Conclusion}

In this study, we demonstrated that $\mathrm{THJ}$ protected myocardial tissue from diabetes-induced injury. The beneficial effects of THJ may be dependent on reducing ROS production and inhibiting NLRP3 activation, thereby blocking excessive secretion of pro-inflammatory cytokines, and further studies indicated that these protective effects were associated with the SIRT1 pathway. Overall, our findings provided insights into the antioxidative stress- and anti-inflammation-related effects and mechanism of THJ in DCM. These findings may further support the clinical use of THJ. However, further studies are required to assess the constituents of $\mathrm{THJ}$ exhibiting efficacy.

\section{Abbreviations}

DCM: Diabetic cardiomyopathy; T2DM: Type 2 diabetes mellitus; ROS: Reactive oxygen species; NLRP: Nucleotide-binding oligomerization domain-like receptor protein; SIRT1: Sirtuin 1; FOXOs: Forkhead transcription factors; TCM: Traditional Chinese medicine; THJ: Taohuajing; STZ: Streptozotocin; TTC: 2, 3, 5-Triphenyltetrazolium chloride; SOD: Superoxide dismutase; MDA: Malondialdehyde; GSH: Glutathione; DHE: Dihydroethidium; FBG: Fasting-blood glucose; LVFS: Left ventricular fractional shorting; LVEF: Left ventricular ejection fraction; LVEDV: Left ventricular end-diastolic volume; LVESV: Left ventricular end-systolic volume; LDH: Lactate dehydrogenase; TNF-a: Tumor necrosis factor-a; CKMB: Creatine kinase-MB; IL-6: Interleukin-6; GSH-Px: Glutathione peroxidase; IL-1a: Interleukin-1a; TEM: Transmission electron microscopy;

PVDF: Polyvinylidene difluoride

\section{Acknowledgments}

None.

\section{Authors' contributions}

$R Y, F L$, and SWW designed the research; RY, YC, and CMW performed the research; $L X, X Z$, and YQD analyzed the data; $R Y$ wrote the manuscript; FL and SWW revised the manuscript. All authors have read and approved the manuscript.

\section{Funding}

This study was funded by the Key Research and Development Plan Projects of Shaanxi Province in China (grant no. 2015SF2-08-01), the Key Research Laboratory of Traditional Chinese Medicine and Natural Medicine in Shaanxi Province (grant no. 2015-164), and the Project of Shaanxi Engineering Technology Research Center (grant no. S2018-ZC-GCZXXY-SF-0005).

\section{Availability of data and materials}

All data used to support the findings of this study are available from the corresponding author upon request.

\section{Ethics approval and consent to participate}

The Ethics Committee for Animal Experimentation of the Air Force Medical University approved the experimental protocol used on laboratory animals (IACUC-20170902). The manuscript does not include any human subjects or human data.

\section{Consent for publication}

Not applicable.

\section{Competing interests}

The authors declare no conflicts of interest.

\section{Author details}

${ }^{1}$ Department of Traditional Chinese Medicine, the First Affiliated Hospital, the Air Force Medical University, 127 Changle West Road, Xi'an 710032, Shanxi, China. ${ }^{2}$ Department of Chinese Materia Medica and Natural Medicines, School of Pharmacy, the Air Force Medical University, 169 Changle West Road, Xi'an 710032, Shanxi, China. ${ }^{3}$ Xi'an Hemoqi Medical Technology Co. Ltd., Xi'an 710075, Shaanxi, China.

Received: 2 April 2020 Accepted: 13 January 2021

Published online: 26 February 2021

\section{References}

1. Shaw JE, Sicree RA, Zimmet PZ. Global estimates of the prevalence of diabetes for 2010 and. Diab Res Clin Pract. 2030;87(1):4-14.

2. Fox CS, Sean C, Sorlie PD, D'Agostino RB, Pencina MJ, Vasan RS, Meigs JB, Daniel L, Savage PJ. Increasing cardiovascular disease burden due to diabetes mellitus: the Framingham heart study. Circulation. 2007;115(12): 1544.

3. Cai L, Kang YJ. Oxidative stress and diabetic cardiomyopathy. Cardiovasc Toxicol. 2001;1(3):181-93.

4. Bugger $\mathrm{H}$, Abel ED. Molecular mechanisms of diabetic cardiomyopathy. Diabetologia. 2014;57(4):660-71.

5. Jia G, Demarco VG, Sowers JR. Insulin resistance and hyperinsulinaemia in diabetic cardiomyopathy. Nat Rev Endocrinol. 2016;12(3):144-53.

6. Pierce GN, Russell JC. Regulation of intracellular Ca2+ in the heart during diabetes. Cardiovasc Res. 1997:34(1):41-7.

7. Xu Z, Patel KP, Lou MF, Rozanski GJ. Up-regulation of $K(+)$ channels in diabetic rat ventricular myocytes by insulin and glutathione. Cardiovasc Res. 2002;53(1):80-8.

8. Ayaz M, Ozdemir S, Ugur M, Vassort G, Turan B. Effects of selenium on altered mechanical and electrical cardiac activities of diabetic rat. Arch Biochem Biophysics. 2004;426(1):83-90.

9. Ayaz M, Turan B. Selenium prevents diabetes-induced alterations in ( $Z n 2+) i$ and metallothionein level of rat heart via restoration of cell redox cycle. Am J Physiol Heart Circ Physiol. 2006;290(3):H1071.

10. Sihem B. E dale a: diabetic cardiomyopathy revisited. Circulation. 2007; 115(25):3213-23.

11. Nishikawa T, Edelstein D, Brownlee M. The missing link: a single unifying mechanism for diabetic complications. Kidney Int. 2000;58(77):S26-30.

12. Ceriello A. New insights on oxidative stress and diabetic complications may lead to a "causal" antioxidant therapy. Diabetes Care. 2003;5(5):1589-96.

13. Hüttemann M. TXNIP links innate host defense mechanisms to oxidative stress and inflammation in retinal Muller glia under chronic hyperglycemia: implications for diabetic retinopathy. Exp Diabetes Res. 2014;2012(1):438238.

14. Beibei L, Bo L, Wenke W, Xiangjuan L, Xiaoman L, Yanfei X, Cheng Z, Yun Z, Mingxiang Z, Fengshuang A. Rosuvastatin alleviates diabetic cardiomyopathy by inhibiting NLRP3 inflammasome and MAPK pathways in a type 2 diabetes rat model. Cardiovascular Drugs Therap. 2014;28(1):33-43.

15. Silvia AL, Ureña-Peralta JR, Maria José MB, ODLC J, Consuelo G. Role of mitochondria ROS generation in ethanol-induced NLRP3 inflammasome activation and cell death in astroglial cells. Front Cell Neurosci. 2014;8(8):216

16. Imai S, Armstrong CM, Kaeberlein M, Guarente L. Transcriptional silencing and longevity protein Sir2 is an NAD-dependent histone deacetylase. Nature 2000, 403(6771):795-800.

17. Chung S, Yao H, Caito S, Hwang JW, Arunachalam G, Rahman I. Regulation of SIRT1 in cellular functions: role of polyphenols. Arch Biochem Biophysics. 2010:501(1):79-90.

18. Anne B, Sweeney LB, J Fitzhugh S, Chua KF, Greer PL, Yingxi L, Hien T, Ross SE, Raul M, Cohen HY. Stress-dependent regulation of FOXO transcription factors by the SIRT1 deacetylase. Science. 2004;303(5666):2011-5.

19. Kops GJPL, Dansen TB, Polderman PE, Ingrid S, Wirtz KWA, Coffer PJ, Ting-T $\mathrm{H}$, Bos JL, Medema RH, Burgering BMT. Forkhead transcription factor FOXO3a protects quiescent cells from oxidative stress. Nature. 2002; 419(6904):316-21.

20. Salminen A, Ojala J, Huuskonen J, Kauppinen A, Suuronen T, Kaarniranta K. Interaction of aging-associated signaling cascades: inhibition of NF-KB signaling by longevity factors FoxOs and SIRT1. Cell Mol Life Sci. 2008;65(78):1049-58.

21. Fusco R, Scuto M, Cordaro M, D'Amico R, Gugliandolo E, Siracusa R, Peritore AF, Crupi R, Impellizzeri D, Cuzzocrea S, et al. N-PalmitoylethanolamideOxazoline Protects against Middle Cerebral Artery Occlusion Injury in 
Diabetic Rats by Regulating the SIRT1 Pathway. Int J Mol Sci. 2019;20(19): 4845. https://doi.org/10.3390/ijms20194845.

22. Zhou X, Seto SW, Chang D, Kiat H, Razmovski-Naumovski V, Chan K, Bensoussan A. Synergistic Effects of Chinese Herbal Medicine: A Comprehensive Review of Methodology and Current Research. Front Pharmacol. 2016;7:201.

23. Pan S-Y, Litscher G, Gao S-H, Zhou S-F, Yu Z-L, Chen H-Q, Zhang S-F, Tang M-K, Sun J-N, Ko K-M. Historical perspective of traditional indigenous medical practices: the current renaissance and conservation of herbal resources. Evid Based Complement Alternat Med. 2014;2014:525340.

24. Liu L, Duan JA, Tang Y, Guo J, Yang N, Ma H, Shi X. Taoren-Honghua herb pair and its main components promoting blood circulation through influencing on hemorheology, plasma coagulation and platelet aggregation. J Ethnopharmacol. 2012;139(2):381-7. https://doi.org/10.1016/j. jep.2011.11.016. Epub 2011 Nov 20. PMID: 22123200.

25. Xi S, Yue L, Shi M, Peng Y, Xu Y, Wang X, Li Q, Kang Z, Li H, Wang Y. The effects of Taoren-Honghua herb pair on pathological microvessel and angiogenesis-associated signaling pathway in mice model of CCl4-induced chronic liver disease. Evid Based Complement Alternat Med. 2016;2016: 2974256.

26. Tu Y, Xue Y, Guo D, Sun L, Guo M. Carthami flos: a review of its ethnopharmacology, pharmacology and clinical applications. Rev Bras. 2015; 25(5):553-66.

27. Zhou X, Siu W-S, Fung C-H, Cheng L, Wong C-W, Zhang C, Liu C-L, Kwok HF, Lau C-P, Wat E, et al. Pro-angiogenic effects of Carthami Flos whole extract in human microvascular endothelial cells in vitro and in zebrafish in vivo. Phytomedicine. 2014;21(11):1256-63.

28. Yu Z, Guotao L. Zengzhen L, al e: effect of peach kernel on protein kinase B signalling pathway in diabetic macrovascular fibrosis rats. Chinese J Integrative Surg. 2019;25(1):61-6.

29. Wang Y, Qin S, Pen G, Chen D, Han C, Miao C, Lu B, Su C, Feng S, Li $W$. Original research: potential ocular protection and dynamic observation of Polygonatum sibiricum polysaccharide against streptozocin-induced diabetic rats' model. Experimental Biol Med. 2017; 242(1):92-101.

30. Chen T, Wang G, Fu T, Zhang Z. The protective effect of Polygonatum sibiricum polysaccharide on myocardial inflammation in type Idiabetic rats. Pharmacol Clin Chin Materia Medica. 2015.

31. Cui X, Wang S, Cao H, Guo H, Li Y, Xu F, Zheng M, Xi X, Han C. A review: the bioactivities and pharmacological applications of Polygonatum sibiricum polysaccharides. Molecules. 2018;23(5):1170.

32. Lu JM, Wang YF, Yan HL, Pei L, Jie Y. Antidiabetic effect of total saponins from Polygonatum kingianum in streptozotocin-induced daibetic rats. J Ethnopharmacol. 2015;179:291-300.

33. Li YY, Deng HB, Wang R, Wu SR. Regulation of polygonati polysaccharide on expression of glycosylated end-product receptor mRNA in cardiac and renal tissues of diabetic mice. Chin J Clin Rehab. 2005;9(47):177-9.

34. Li K, Zhai M, Jiang L, Song F, Zhang B, Li J, Li H, Li B, Xia L, Xu L, et al. Tetrahydrocurcumin ameliorates diabetic cardiomyopathy by attenuating high glucose-induced oxidative stress and fibrosis via activating the SIRT1 pathway. Oxidative Med Cell Longev. 2019;2019:6746907.

35. Fuping Z, Wuping L, Linhua W, Chengxi P, Fuqiang Z, Yi Z, Aijun W. TaoHong-Si-Wu decoction reduces ischemia reperfusion rat myoblast cells calcium overloading and inflammation through the Wnt/IP3R/CAMKII pathway. J Cell Biochem. 2019;120(8):13095-106.

36. Li L, Yang N, Nin L, Zhao Z, Chen L, Yu J, Jiang Z, Zhong Z, Zeng D, Qi H, et al. Chinese herbal medicine formula tao hong si wu decoction protects against cerebral ischemia-reperfusion injury via PI3K/Akt and the Nrf2 signaling pathway. J Nat Med. 2015;69(1):76-85.

37. Zhang B, Zhai M, Li B, Liu Z, Li K, Jiang L, Zhang M, Yi W, Yang J, Yi D. Honokiol Ameliorates Myocardial Ischemia/Reperfusion Injury in Type 1 Diabetic Rats by Reducing Oxidative Stress and Apoptosis through Activating the SIRT1-Nrf2 Signaling Pathway. Oxidative Medicine and Cellular Longevity,2018,(2018-2-20). 2018;2018:1-16.

38. Zhai M, Liu Z, Zhang B, Jing L, Li B, Li K, Chen X, Zhang M, Yu B, Ren K. Melatonin protects against the pathological cardiac hypertrophy induced by transverse aortic constriction through activating PGC- $1 \hat{T}^{2}$ : in vivo and in vitro studies. J Pineal Res. 2017;63(3):e12433.

39. Jialin D, Ying Y, Guo W, Jia C, Enhu Z, Yue G, Jiajia Y, Chao G, Yanrong Z, Fei M. Chikusetsu saponin IVa confers cardioprotection via SIRT1/ERK1/2 and Homer1a pathway. Sci Rep. 2015;5:18123.
40. Aneja A, Tang WHW, Bansilal S, Garcia MJ, Farkouh ME. Diabetic cardiomyopathy: insights into pathogenesis, diagnostic challenges, and therapeutic options. Am J Med. 2012;121(9):748-57.

41. Schultheiss H-P, Fairweather D, Caforio ALP, Escher F, Hershberger RE, Lipshultz SE, Liu PP, Matsumori A, Mazzanti A, McMurray J, et al. Dilated cardiomyopathy. Nat Rev Dis Primers. 2019;5(1):32.

42. Duan J, Wei G, Guo C, Cui J, Yan J, Yin Y, Guan Y, Weng Y, Zhu Y, Wu XJ. Aralia taibaiensis Protects Cardiac Myocytes against High Glucose-Induced Oxidative Stress and Apoptosis. Am J Chin Med. 2015;43(6):1159-75.

43. Tong $X-L$, Dong $L$, Chen $L$, Zhen Z. Treatment of diabetes using traditional Chinese medicine: past. Present Future. 2012;40(05):877-86.

44. Hsu P-C, Tsai Y-T, Lai J-N, Wu C-T, Lin S-K, Huang C-Y. Integrating traditional Chinese medicine healthcare into diabetes care by reducing the risk of developing kidney failure among type 2 diabetic patients: a population-based case control study. J Ethnopharmacol. 2014;156:358-64.

45. Luo Z-R, Li H, Xiao Z-X, Shao S-J, Zhao T-T, Zhao Y, Mou F-F, Yu B, Guo $H-D$. Taohong Siwu decoction exerts a beneficial effect on cardiac function by possibly improving the microenvironment and decreasing mitochondrial fission after myocardial infarction. Cardiol Res Pract. 2019; 2019:5198278.

46. Zhou J, Yang D, Zhou SH, Wang JP, Liu YS, Wang SL. Clinical Efficacy and Safety of Bathing with Chinese Medicine Taohong Siwu Decoction (桃红四 物汤) for Treatment of Diffuse Cutaneous Systemic Sclerosis: A Randomized Placebo-Controlled Trial. Chin J Integr Med. 2018;24(3):185-192. https://doi. org/10.1007/s11655-017-2954-2. Epub 2017 Feb 15. PMID: 28197938.

47. Zhang Y, Wang J, Zuo C, Chen W, Zhu Q, Guo D, Wu H, Wang H, Peng D, Han L. Protective effect of Taohong Siwu decoction on abnormal uterine bleeding induced by incomplete medical abortion in rats during early pregnancy. Chem Pharm Bull. 2018;66(7):708-13.

48. Yen T-L, Ong E-T, Lin K-H, Chang C-C, Jayakumar T, Lin S-C, Fong T-H, Sheu J-R. Potential advantages of Chinese medicine Taohong Siwu decoction (桃 红四物汤) combined with tissue-plasminogen activator for alleviating middle cerebral artery occlusion-induced embolic stroke in rats. Chin J Integrative Med. 2014;1-9. https://doi.org/10.1007/s1 1655-014-1847-x.

49. Fernandez EB. Glucolipotoxicity, resistance to the action of insulin and type 2 diabetes mellitus. Anales De La Real Academia Nacional De Medicina. 2007;124(3):547.

50. Bell DSH. Diabetes: a cardiac condition manifesting as hyperglycemia. Endocrine Practice Official Journal of the American College of Endocrinology \& the American Association of Clinical Endocrinologists. 2008;14(7):924-32.

51. Yan Y, Wei J, Spinetti T, Tardivel A, Castillo R, Bourquin C, Guarda G, Tian Z, Tschopp J, Zhou R. Omega-3 fatty acids prevent inflammation and metabolic disorder through inhibition of NLRP3 Inflammasome activation. 全国免疫学学术大会. 2012;2012.

52. Vishwa Deep D. Nlrp3 inflammasome activation in type 2 diabetes: is it clinically relevant? Diabetes. 2013;62(1):22-4.

53. Shengyi S, Sheng X, Yewei J, Sander K, Ling Q. The ATP-P2X7 signaling axis is dispensable for obesity-associated inflammasome activation in adipose tissue. Diabetes. 2012;61(6):1471-8.

54. Lerner AG, John-Paul U, Praveen PVK, Rajarshi G, Yoshimi N, Aeid I, Sarah S, Vinh N, Backes BJ, Myriam H. IRE1a induces thioredoxininteracting protein to activate the NLRP3 inflammasome and promote programmed cell death under irremediable ER stress. Cell Metab. 2012; 16(2):250-64

55. Fusco R, Gugliandolo E, Biundo F, Campolo M, Di Paola R, Cuzzocrea S. Inhibition of inflammasome activation improves lung acute injury induced by carrageenan in a mouse model of pleurisy. FASEB J. 2017; 31(8):3497-511.

56. Schroder K, Tschopp J. The Inflammasomes. Cell. 2010;140(6):821-32.

57. Franchi L, Muñozplanillo R, Núñez G. Sensing and reacting to microbes through the inflammasomes. Nat Immunol. 2012;13(4):325-32.

58. Duan J, Cui J, Zheng H, Xi M, Guo C, Weng Y, Yin Y, Wei G, Cao J, Wang $Y$, et al. Aralia taibaiensis protects against I/R-induced brain cell injury through the Akt/SIRT1/FOXO3a pathway. Oxidative Med Cell Longev. 2019;2019:1-18.

59. Hien T, Anne B, Grenier JM, Datta SR, Fornace AJ, Distefano PS, Chiang LW, Greenberg ME. DNA repair pathway stimulated by the forkhead transcription factor FOXO3a through the Gadd45 protein. Science. 2002: 296(5567):530-4. 
60. Yoko FH, Kiyomi YA, Tsutomu O, Kyoji I, Noboru M. FOXO forkhead transcription factors induce $\mathrm{G}$ (2)-M checkpoint in response to oxidative stress. J Biol Chem. 2002;277(30):26729-32.

61. Ryusuke H, Atsushi K, Hori YS, Katsuki O, Nobutaka W, Azusa O, Hiroki H, Yoshiyuki H. Differential cell-protective function of two resveratrol (trans3,5,4'-trihydroxystilbene) glucosides against oxidative stress. J Pharmacol Experimental Therap. 2013;344(1):124-32.

62. Li Y, Yang $X$, He Y, Wang W, Zhang J, Zhang W, Jing T, Wang B, Lin R. Negative regulation of NLRP3 inflammasome by SIRT1 in vascular endothelial cells. Immunobiology. 2016;222(3):552-61.

\section{Publisher's Note}

Springer Nature remains neutral with regard to jurisdictional claims in published maps and institutional affiliations.

Ready to submit your research? Choose BMC and benefit from:

- fast, convenient online submission

- thorough peer review by experienced researchers in your field

- rapid publication on acceptance

- support for research data, including large and complex data types

- gold Open Access which fosters wider collaboration and increased citations

- maximum visibility for your research: over $100 \mathrm{M}$ website views per year

At BMC, research is always in progress.

Learn more biomedcentral.com/submissions 